\title{
Acceleration of the FINE/Turbo CFD Solver in a Heterogeneous Environment with OpenACC Directives
}

\author{
David Gutzwiller * \\ NUMECA USA \\ 1044 Larkin Street \\ San Francisco, California, \\ USA \\ david.gutzwiller \\ @numeca.com
}

\author{
Dr. Ravi Srinivasan ${ }^{\dagger}$ \\ Research and Development, \\ Seattle Technology Center \\ 11808 Northup Way, Suite \\ W-190 \\ Bellevue, Washington, USA \\ rsrinivasan \\ @dresser-rand.com
}

\author{
Dr. Alain Demeulenaere ${ }^{\ddagger}$ \\ NUMECA USA \\ 1044 Larkin Street \\ San Francisco, California, \\ USA \\ alain.demeulenaere \\ @numeca.com
}

\begin{abstract}
Adapting legacy applications for use in a modern heterogeneous environment is a serious challenge for an industrial software vendor (ISV). The adaptation of the NUMECA FINE/Turbo computational fluid dynamics (CFD) solver for accelerated CPU/GPU execution is presented. An incremental instrumentation with OpenACC directives has been used to obtain a global solver acceleration greater than $2 \mathrm{X}$ on the OLCF Titan supercomputer. The implementation principals and procedures presented in this paper constitute one successful path towards obtaining meaningful heterogeneous performance with a legacy application. The presented approach minimizes risk and developer-hour cost, making it particularly attractive for ISVs.
\end{abstract}

\section{Categories and Subject Descriptors}

C.1 [Processor Architectures]: Other Architecture-Heterogeneous (hybrid) systems;

D.2 [Software Engineering]: Distribution, Maintenance, and Enhancement;

D.3 [Programming Languages]: Miscellaneous

\section{General Terms}

GPU Acceleration

\section{Keywords}

Heterogeneous, Turbomachinery,OpenACC,CFD,Directives

\author{
*NUMECA USA: Head of HPC. \\ ${ }^{\dagger}$ Dresser-Rand: Aero/Thermodynamics Engineer.

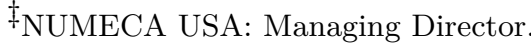

Permission to make digital or hard copies of all or part of this work for personal or classroom use is granted without fee provided that copies are not made or distributed for profit or commercial advantage and that copies bear this notice and the full citation on the first page. To copy otherwise, to republish, to post on servers or to redistribute to lists, requires prior specific permission and/or a fee.

WACCPD2015 November 15-20, 2015, Austin, TX, USA

Copyright 2015 ACM ISBN 978-1-4503-4014-4/15/11 ...\$15.00

DOI: http://dx.doi.org/10.1145/2832105.2832114.

\section{INTRODUCTION}

Over the last decade heterogeneous cluster architectures have evolved from an exotic concept to a common high performance computing (HPC) solution, deployed on systems ranging in size from a few nodes to top 500 supercomputers with thousands of nodes. In response to this rapid paradigm shift software vendors must now address a difficult question: what is the quickest and safest way to update legacy applications for heterogeneous execution? Whatever the solution, the migration to heterogeneous architectures should not negatively impact performance on traditional homogenous systems or excessively complicate the maintainence and continued development of the application. There is no easy answer to this question, and no technology is suitable for all applications. However, a directive based approach is a very quick and attractive option because it does not require the rewriting and maintenance of separate code branches for homogenous and heterogeneous architectures.

This paper describes the successful adaptation of the NUMECA FINE/Turbo CFD solver for execution on a heterogeneous CPU/GPU architecture using an incremental acceleration with OpenACC directives. A brief overview of the pre-existing FINE/Turbo solver structure and distributed parallel model is presented along with a discussion of heterogeneous CPU/GPU implications. The time, manpower, and logistics contraints that NUMECA and other ISVs must consider when updating legacy applications is also be presented. Further discussion delves into the details of the FINE/Turbo adaptation. The typical properties of an OpenACC accelerated routine, memory handling and data transfer model, and the implementation of a heterogeneous auto-tuning class are described in detail.

Finally, a representative example computation is shown. The computation is a high resolution supersonic inlet/isolator simulation solved on the Oak Ridge Leadership Computing Facility (OLCF) Titan Cray XK7 supercomputer. This real-world example shows a greater than $2 \mathrm{X}$ speedup in time to solution.

\section{NUMECA FINE/TURBO CFD SOLVER}

FINE/Turbo is a general purpose CFD toolset adapted primarily for high fidelity turbomachinery analysis. The FINE/Turbo flow solver is a legacy toolset consisting of a FORTRAN core wrapped in a $\mathrm{C}$ and $\mathrm{C}++$ interface. The 


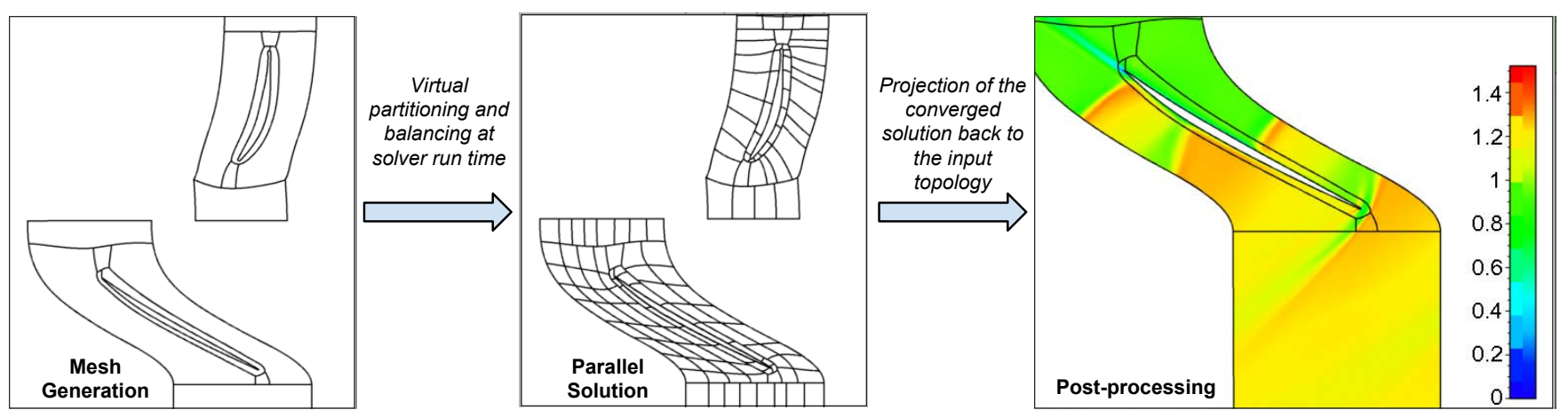

Figure 1: FINE/Turbo Virtual Partitioning

solver is a three dimensional, density based, structured, multi block Navier-Stokes code using a finite volume method; central difference space discretization is employed for the spatial discretization with Jameson type artificial dissipation [5]. A four-stage explicit runge-kutta scheme is applied for the temporal discretization, multi-grid methods, local timestepping and implicit residual smoothing are used to obtain very quick convergence. Available solver modules allow steady and time-accurate solutions with a wide range of fluid models, turbulence models, and arbitrary mesh topologies with matching and non-conformal block boundary conditions.

\subsection{Pre-existing Parallel Model}

The FINE/Turbo parallel model is based upon the distribution of the structured blocks among an arbitrary number of MPI processes. The multi-grid methodology requires that each structured block be coarsened by node skipping in each of the $\mathrm{I} / \mathrm{J} / \mathrm{K}$ directions, with all coarsened blocks hosted on the same MPI process as the associated fine block. This model has the obvious limitation that the solution of each structured block occurs on no more than one MPI process, which effectively limits the parallelism to the number of blocks in the mesh. To resolve this limitation and allow effective use of a homogenous HPC system, a virtual partitioning algorithm has been implemented into FINE/Turbo, further dividing and balancing the grid over an arbitrary number of processes at run time [4]. The virtual nature of the partitioning algorithm makes the entire process transparent to the end user, who interacts with the model only through a simple unpartitioned topology. Figure 1 elaborates on this procedure.

On a traditional homogenous cluster, this scheme has allowed near-linear scalability with thousands of MPI processes and quick solution time for meshes with billions of points $[2,3]$. A representative speedup curve is shown in Figure 2.

\subsection{Pre-existing Memory Model}

FINE/Turbo uses a legacy FORTRAN memory model in which monolithic real and integer vectors are allocated at the start of the computation on each process, with the size of the allocation being the same on all processes. Internal memory management functions are used to push and pop temporary memory space on the end of the vectors, mimicking dynamic memory without expensive allocation

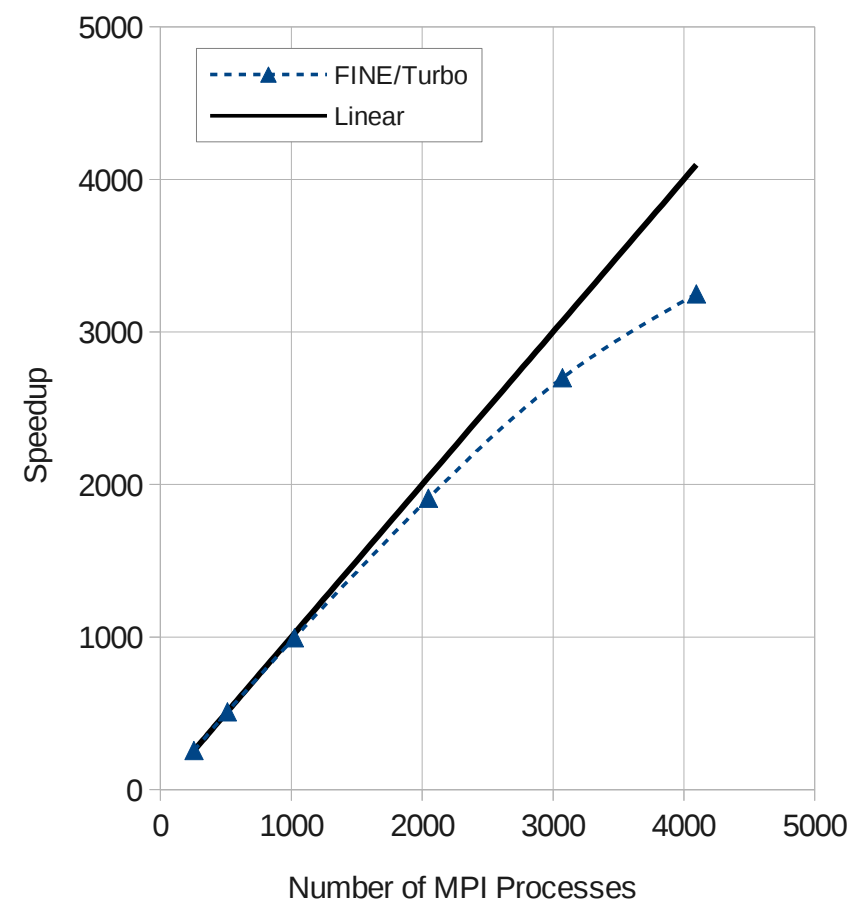

Figure 2: FINE/Turbo Distributed Parallel Scalability 300 million cell steady computation, 3 multi-grid levels.

operations. To ensure efficient memory scalability all mesh dependent data is partitioned among the processes, while a small amount of redundant topological data is stored on all processes.

\subsection{Heterogeneous CPU/GPU Implications}

Changing the underlying structure of FINE/Turbo would be very expensive in terms of developer-hours. Thus, from the start of this work, it was known that the support of heterogeneous architectures would have to fit within the existing distributed parallel model. As shown in section 3, OpenACC is well suited to this task. However, it is prudent to first summarize the impact that the existing FINE/Turbo structure and parallel model has on the adaption for GPU acceleration. 
- Block Size, Multi-grid, and Exposed Parallelism: The multi-block structured nature of the FINE/Turbo solver limits the number of threads that may be safely spawned for parallel execution on a GPU accelerator. In general, the maximum number of threads that may be spawned in a particular kernel is limited to the number of cells in the 3D block. Naturally, the available number of threads is higher when solving the fine grid as opposed to the coarse grids. This is important to note because a typical FINE/Turbo virtually partitioned mesh contains blocks of varying size with multiple coarse grid levels. Depending on the hardware, there is certainly a cutoff block size at which there is not enough exposed parallelism to justify the use of the GPU instead of the main CPU.

- GPU Memory and Data Transfer: Most current heterogeneous CPU/GPU systems utilize separate memory spaces for the CPU and GPU, with the GPU memory typically much smaller than the main CPU memory. An implication of this is that frequent memory allocations and transfers to/from the main memory and the GPU memory around accelerated kernels are necessary. Frequently transferring the entirety of the monolithic real and integer vectors is not efficient, so the data transfer model must allow efficient handling of subarrays.

- Multiple MPI Processes per GPU: The distributed parallel model employed by FINE/Turbo is most effective when all CPU cores on a node are utilized. Typical CPU/GPU nodes have a multi-core CPU with upwards of 16 cores and one or two GPUs. The implication is that each GPU accelerates and transfers data with multiple MPI processes concurrently.

\section{ACCELERATION WITH OPENACC}

\subsection{Development Goals and Constraints}

The main goal of this project is to obtain a large GPU speedup on the target OLCF Titan supercomputer with a minimal amount of development time. As an ISV, NUMECA must operate with additional goals and contraints. First, FINE/Turbo is developed by a team of engineers working simultaneously on various parts of the code, with all changes coordinated with an industry standard versioning system, SVN. It is critical that the chosen acceleration technology allows incremental improvements to the solver code so as to not disrupt other ongoing development work. Also, the FINE/Turbo solver is in use at many customer sites that still utilize traditional homogenous CPU-only hardware, and the adaptation for heterogeneous execution must not negatively impact the existing performance. The accelerated solver must be easily portable on the major competing accelerator technologies including NVIDIA GPUs, AMD GPUs, and Intel Xeon Phi coprocessors. Finally, the accelerated solver must not yield different results due to the chosen acceleration hardware or lack thereof.

\subsection{Programming Technology}

Heterogeneous CPU/GPU architectures are still a relatively new technology, and not all of the available development technologies have reached sufficient levels of matu- rity for use by an ISV. Furthermore, not all available technologies meet the additional contraints listed in section 3.1. Rewriting FINE/Turbo in a low level GPU programming language like OpenCL or CUDA is not a very attractive option because it necessitates wholesale changes to the low level routines resulting in duplicate sources which must be maintained. Another potential option would be to utilize GPU-accelerated libraries such as CUBLAS [6]. At first glance this seems like a very attractive option because it is a very high level option that requires neither a deep understanding of the target hardware or the learning of a new programming language. However, there are very few places in the FINE/Turbo solver where the use of a "drop-in" library would be appropriate and the resulting GPU acceleration would be very limited.

A third option exists in the form of compiler directives, either OpenMP4 or OpenACC directives [10, 9]. This approach allows a level of control between a low level GPU programming language and GPU accelerated drop-in libraries. This is the most appealing option for acceleration of a legacy industrial CFD solver such as FINE/Turbo. OpenMP4 and OpenACC are similar in their intended usage and both look promising, but in practice neither is completely mature yet. At the start of this work a brief survey identified Ope$\mathrm{nACC}$ as being more feature-rich and robust for the target CPU+NVIDIA GPU architecture [7]. While OpenACC was the chosen application programming interface for this project, it is recommended to periodically review the available options as they mature. OpenACC offers:

- Minimal changes to the existing CPU code, resulting in little increase in long term code maintenance.

- Memory management and data locality control flexible enough for the legacy FINE/Turbo memory model.

- A high level of support from well respected compiler groups such as PGI and the GNU Project.

- Automatic support and optimization for a wide range of target hardware configurations, depending on the chosen compiler.

\subsection{Heterogeneous Programming Model}

The adaptation of FINE/Turbo for CPU/GPU execution was achieved through the acceleration of high cost, end of call tree routines with OpenACC directives. The FINE/Turbo solver call tree is arranged in such a way that most of the parallelizable math is contained within the end of call tree routines. An initial profile of the solver found that no individual routine accounts for more than $20 \%$ of the solver execution time on CPU, and dozens of routines account for more than $1 \%$ of the execution time. From this flat profile it was known that many routines would need to be instrumented before a large global solver speedup would be observed. However, incremental speedups were expected, with each instrumented routine slightly improving the observed speedup.

\subsection{Properties of a Targeted Routine}

As mentioned in section 3.3, the FINE/Turbo execution time is distributed among dozens of end of call tree routines. However, not all of these routines are well suited for efficient threaded execution. Routines that were targeted for acceleration have the following properites: 
- Large amounts of thread-safe math contained within 3D loops, or 2D planar loops which could easily be converted to $3 \mathrm{D}$ loops.

- Minimal code branching and logic.

- Few nested function calls, or function calls which may be replaced with intrinsics.

- Deterministic algorithms with simple subarray inputs and outputs.

With these properties in mind, the following sets of routines were targeted for acceleration with OpenACC directives.

- Viscous flux calculation.

- Convective flux calculation.

- Artificial dissipation calculation.

- Implicit residual smoothing.

- Source term calculation.

- CPUBooster convergence acceleration [8].

Depending on the project configuration, it is known from the solver profiling that these routines account for $50 \%-75 \%$ of the total solver execution time. Therefore, it is known that the theoretical solver speedup ignoring data transfer time is limited to $2 \mathrm{X}-4 \mathrm{X}$.

\subsection{Memory Handling and Data Transfer}

The amount of on-board memory is a limiting property of modern GPUs. High end supercomputers, such as the the OLCF Titan system, utilize GPUs with upwards of 6-12 gb of on board memory. Standalone workstations and consumer level hardware may have GPUs with only 1 or $2 \mathrm{gb}$ of memory. CFD solvers are typically quite memory intensive, and a FINE/Turbo steady simulation requires approximately 500 mb of memory for every million cells. Typical workstation size computations utilize meshes with a few million cells, while larger computations on clusters may have upwards of a billion cells. It can be assumed that current high end GPUs have sufficient memory for most FINE/Turbo computations, but for certain configurations it could still be a limitation. Given the existing FINE/Turbo memory model the developer is left with two implementation options:

- High GPU Performance Mode: Permanently allocate the entire integer and work vectors on the GPU for all associated MPI processes at the start of the computation. This approach minimizes data transfer time as all static data, such as grid coordinates, are always present on the GPU. Expensive memory allocation time is also minimized because all necessary temporary space is pre-allocated in the real vector and managed by FINE/Turbo. In this mode data transfer is limited to updating specific subarrays at the entrance and exit of data regions lower in the call tree. In summary: this option favors improved data locality at the cost of extra GPU memory usage.
- Low GPU Memory Mode: Temporarily allocate the necessary GPU memory at the entrance of each accelerated region. All necessary quantities must be allocated and updated on the GPU at the entrance of data regions deep in the call tree, and all memory deallocated at the exit. This option utilizes significantly less memory as only the specific quantities for the current block are allocated on the GPU. In summary: this option improves GPU memory usage at the cost of poor data locality.

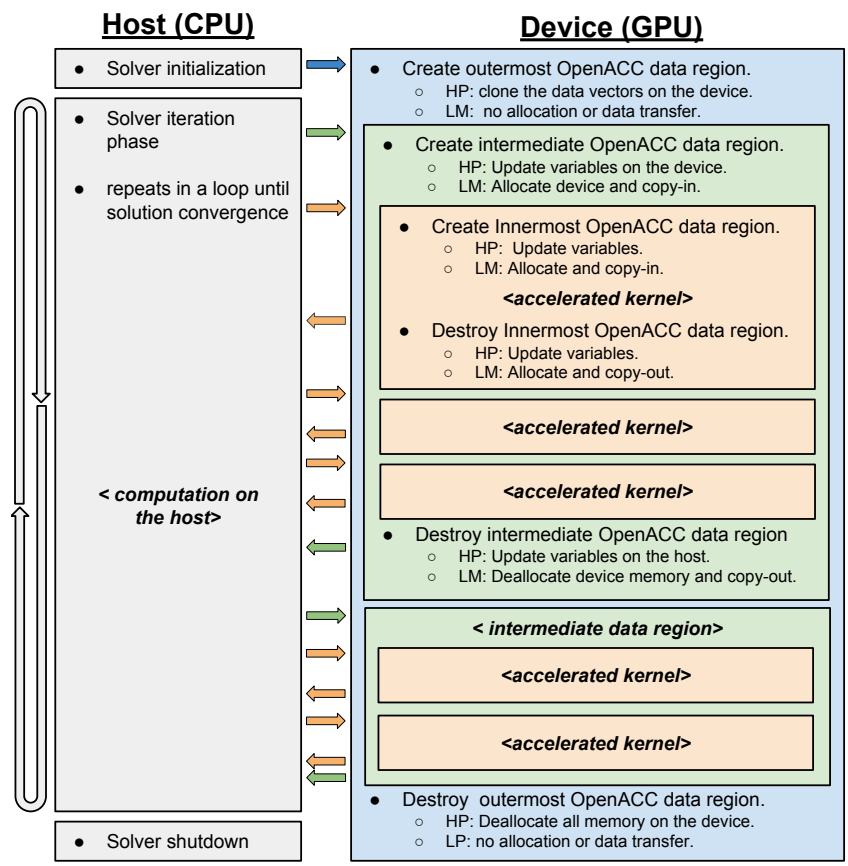

Figure 3: FINE/Turbo OpenACC Memory Management and Data Transfer. High GPU Performance mode (HP) and Low GPU Memory mode (LM).

Neither option above listed above is ideal for all potential customer hardware, and it is worthwhile for an ISV to implement both. Fortunately, the OpenACC memory management directives are flexible enough to support both options with a single implementation. As shown in Figure 3 , a top-level OpenACC data region is created during the solver initialization to handle the conditional allocation and transfer of the integer and work vectors. In each of the data regions lower in the call tree the present_or_create, present_or_copyin, and update directives are used. The present_or_<action $>$ directives first check the device memory table for the presence of the requested subarray. The $<$ action $>$ is then only taken if the requested subarray is not present in device memory. This clever feature of OpenACC allows very useful systems of conditional nested data regions without complicated logic or code branching. For example, if on a particular piece of hardware there is insufficient onboard memory to create the outermost data region, it may still be possible to host one or more intermediate data regions. These intermediate data regions are important because they help minimize redundant data transfers in the innermost data regions. The OpenACC data management directives allow this without any extra development effort. 
The relative GPU memory usage of the two memory modes is primarily determined by the size and number of virtually partitioned blocks hosted on each solver process. For most FINE/Turbo computations the Low GPU Memory Mode has a GPU memory footprint $1 / 3$ that of the High GPU Memory Mode. Which memory mode to use is decided by the end-user with a simple run time flag.

\subsection{Race Condition Avoidance}

Some of the routines targeted for acceleration were not entirely thread safe, and extra care was needed to obtain safe, fast performance on both the CPU and GPU. A simplified example of a triple nested loop is shown below:

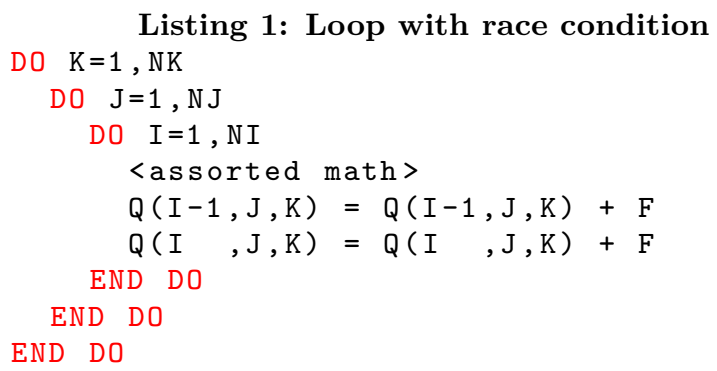

This loop is problematic; the concurrent update of both the I and I-1 cells is not likely to lead to a solver crash or a deadlock condition, but it could lead to incorrect and nondeterministic results. Completely refactoring all of the targeted routines would be time consuming and risky. Alternatively, a simplified coloring approach on the innermost I loop resolves the race condition with no changes to the underlying algorithms [11]. However, this leads to another problem as the memory access when solved on a traditional CPU is no longer stride 1 , and performance is penalized. Thus, the following conditional coloring was implemented. When executed on a traditional CPU the NCOLOR variable is set to 1 , and the subsequent loops degenerate to a standard triple nested loop. NCOLOR is set to 2 when executed on a GPU, and the triple nested loop is launched as two sequential parallel kernels, one with $\mathrm{I}=1,3,5, \ldots, \mathrm{NI}$ and one with $\mathrm{I}=2,4,6, \ldots, \mathrm{NI}$.

Listing 2: Thread-safe loop with coloring IF (IGPU.EQ.1)

$\mathrm{NCOLOR}=2$

ELSE

NCOLOR $=1$

\section{ENDIF}

! \$acc parallel loop if(IGPU.EQ.1) gang

DO $\mathrm{K}=1, \mathrm{NK}$

! \$acc loop worker

DO $\mathrm{J}=1, \mathrm{~N} \mathrm{~J}$

DO COLOR $=0,($ NCOLOR -1$)$

! \$acc loop vector

DO $\mathrm{I}=(1+\mathrm{COLOR}), \mathrm{NI}, \mathrm{NCOLOR}$

<assorted math>

$Q(I-1, J, K)=Q(I-1, J, K)+F$

$Q(I, J, K)=Q(I, J, K)+F$

END DO

END DO

END DO

END DO

\subsection{Acceleration Auto-Tuning}

When developing a general purpose application user friendliness cannot be ignored. The GPU acceleration presented in this paper presents a particularly difficult problem because the observed GPU speedup of an accelerated kernel depends on many factors:

- Speed of the CPU.

- Speed of the GPU.

- Latency and bandwidth of the CPU/GPU data pipeline.

- Shape and size of the current block.

- Multi-grid level of the current block.

- Amount and type of operations in the current kernel.

- Number of MPI processes concurrently launching kernels on the GPU

With all of these factors in mind, it is impossible to predict which accelerated kernels will exhibit a meaningful speedup on the GPU. Furthermore, it is unreasonable to expect the end-user to tune the implementation for their particular hardware. A more elegant solution than a user-controlled on/off switch is needed. This solution should be automatic and should minimize the risk associated with activating the GPU acceleration. The use of the GPU acceleration should never result in a solver slowdown, even when used with older or low-end GPU hardware.

To achieve this goal an acceleration management module has been created, the AccManager class. In summary: this class utilizes a std::map to associate timer and execution flag data with each accelerated kernel for each virtually partitioned block and multigrid level. At solver run time, an instance of this class is created on each MPI process, monitoring the execution time of the accelerated kernels for the blocks hosted on that process. During the first two solver iterations the AccManager performs trial executions of each kernel on the CPU and GPU. From the stored timer data the AccManager class determines which kernels, virtually partitioned blocks, and grid levels should be offloaded to the GPU and which kernels are better left on the main CPU. This approach has obvious benefits for the end-user as it allows automatic tuning of the solver with a single user toggle. The AccManager also aids in the development process as it removes some of the risk associated with expanding the GPU acceleration to additional solver modules. If current hardware is insufficient to accelerate a particular routine the AccManager automatically determines that the kernels should be executed on the main CPU, removing any chance of an unintended solver slowdown. In practice, the AccManager has been shown to significantly improve the GPU solver speedup relative to simple grid level filtering or a master toggle, as shown in section 3.8.

An in-depth analysis of the AccManager class is outside the scope of this paper, but a discussion of known side effects is warranted. There is one instance of the AccManager per MPI process, with each instance making acceleration decisions independently. Due to the multi-block structured nature of the FINE/Turbo solver it is likely that each process hosts a different number of blocks of different sizes. In this situation the natural staggering of the data transfers 
avoids slowdown from data transfer contention. However, it is also possible that multiple MPI processes host blocks of similar size, resulting in synchronized entry to accelerated kernels and contention in the data transfer. In this situation the perceived kernel acceleration varies significantly on each process even though the accelerated blocks are of similar size. The AccManager on the slower processes may choose to offload the kernels to the CPU. In effect, this can be viewed as a multi-process load-balancing tool between the CPU and GPU, with some processes running primarily on the CPU with improved memory bandwidth and other processes taking advantage of less contention during GPU data transfer. However, if many processes are blocked by data contention it is possible that the independent AccManager instances will determine an overly conservative configuration, with all but one of the processes relegating a particular kernel to the CPU. In this situation a more dynamic load balancing might yield better results.

\subsection{Heterogeneous Results}

The performance of the OpenACC instrumented FINE/Turbo solver is shown in Figure 4. Version 14.7 of the PGI compiler was used for the preparation of the GPU accelerated binary. All of the tests were performed using 16 nodes of the OLCF Titan supercomputer, with 128 MPI processes offloading calculations to 16 NVIDIA Tesla K20 GPUs. Thus, each Tesla concurrently accelerated 8 MPI processes. The test computation is a 67 million cell steady simulation with 3 multigrid levels, the details of which are shown in section 4 .

These results illustrate a few important points. First, the CPU-only performance of the FINE/Turbo solver has not been penalized by the minor code restructuring needed for heterogeneous execution. These results also clearly show the performance benefits of automatic acceleration tuning with the AccManager class. The use of the AccManager along with the "High GPU Performance" mode results in a global speedup of 2.59X, compared to $2.4 \mathrm{X}$ if all of the accelerated kernels are blindly offloaded to the GPU. This impact is much more pronounced when utilizing the "Low GPU Memory" mode, trading poor data locality for much lower GPU memory usage. In this mode many of accelerated kernels experience a significant slowdown due to a large increase in data transfer time, resulting in an application slowdown of nearly 5X. Enabling the AccManager automatically determines the kernels for which a speedup is still possible, and the slowdown is converted to a modest speedup of $1.32 \mathrm{X}$. As expected, the obtained global speedup of $1.32 \mathrm{X}$ with the "Low GPU Memory" mode is much lower than the 2.59X obtained with the "GPU High Performance" mode.

\section{REPRESENTATIVE EXAMPLE}

A supersonic inlet/isolator model based on the experiments of Emami et al.[1] was used to evaluate the impact of the latest GPU porting on the time to solution. The experimental setup was designed to investigate the inlet/isolator performance in a scramjet engine. For the purposes of this test simulation, a single operating point was selected from the available matrix of experimental results. This operating point is labeled Run 140 and executed at maximum back pressure before inlet unstart.

The simulation domain consisted of the inlet ramp, isolator, side walls, cowl and diffuser, shown in Figure 5. A 3D

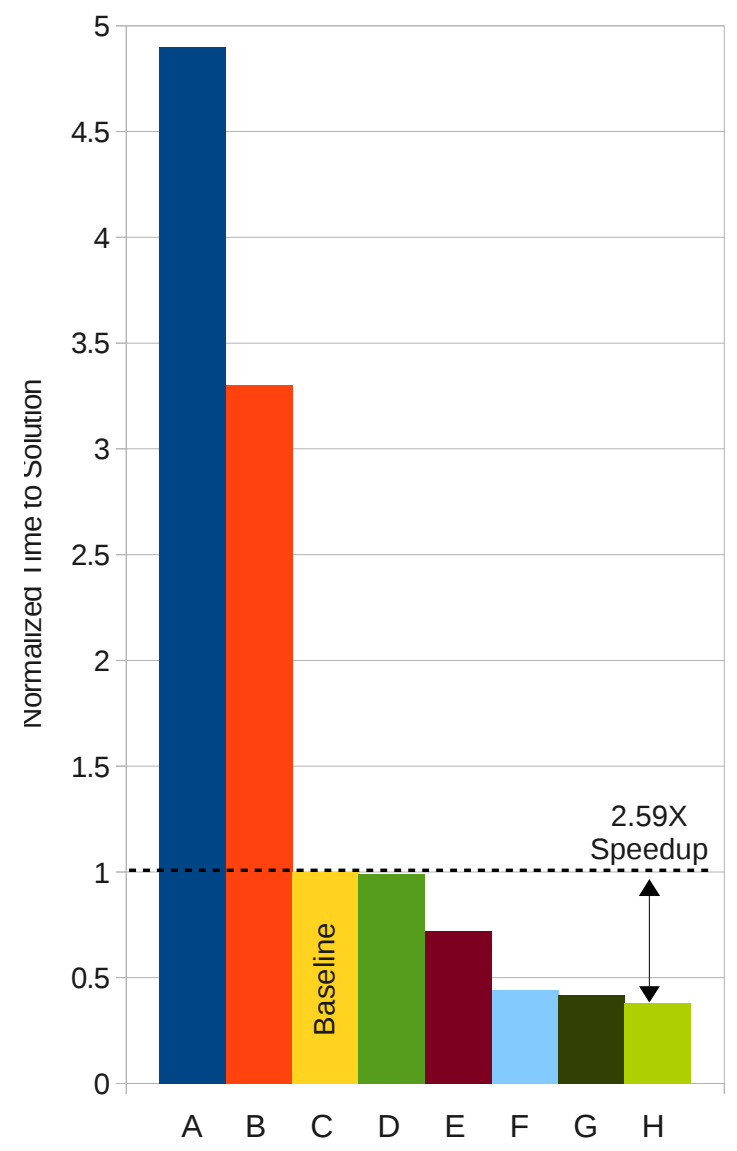

Figure 4: FINE/Turbo OpenACC acceleration. Normalized time to solution. [A] Low GPU memory mode, all kernels accelerated [B] Low GPU memory mode, coarsest grid level on the CPU [C] CPU-only execution time before code modifications [D] CPU-only execution time after code modifications [E] Low GPU memory mode, AccManager enabled [F] High GPU performance mode, all kernels accelerated [G] High GPU performance mode, coarsest grid level on the CPU [H] High GPU performance mode, AccManager enabled.

structured, multi-block grid was generated for the geometry using the NUMECA Interactive Grid Generator (IGG). The grid is comprised of 23 blocks with a total of approximately 67 million grid points, with the largest and smallest block containing approximately 12.5 million and 78 thousand points, respectively.

The simulation was run with freestream boundary conditions at the inlet with a static pressure of $8726 \mathrm{~Pa}$, static temperature of $65 \mathrm{~K}$ and freestream axial velocity of 651.28 $\mathrm{m} / \mathrm{s}$. The domain outlet pressure was set to an average static pressure of $414.49 \mathrm{kPa}$. The cowl outlet boundary values were extrapolated from the interior of the domain. The solution is second order accurate in space and based on the JST scheme. Convergence was accelerated using local time-stepping and by using the CPUBooster module.

Contour plots of axial and streamwise Mach number in the domain are shown in Figure 7. The inlet leading edge 


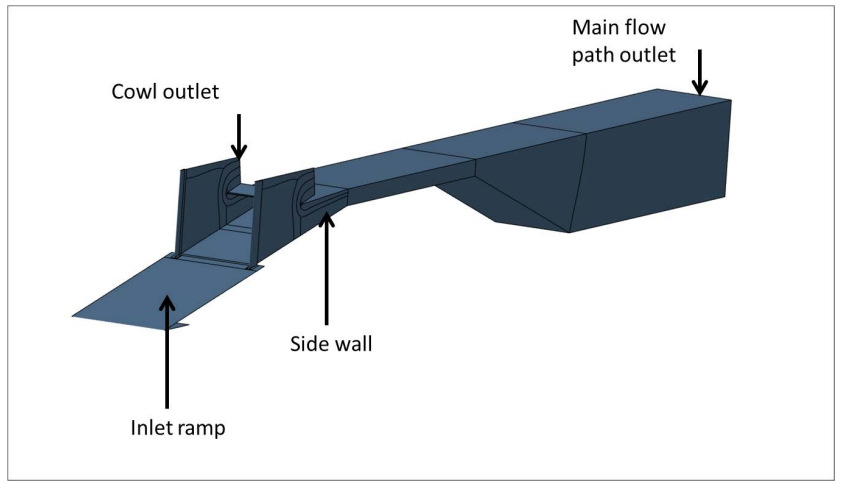

Figure 5: Supersonic Inlet/Isolator Geometry

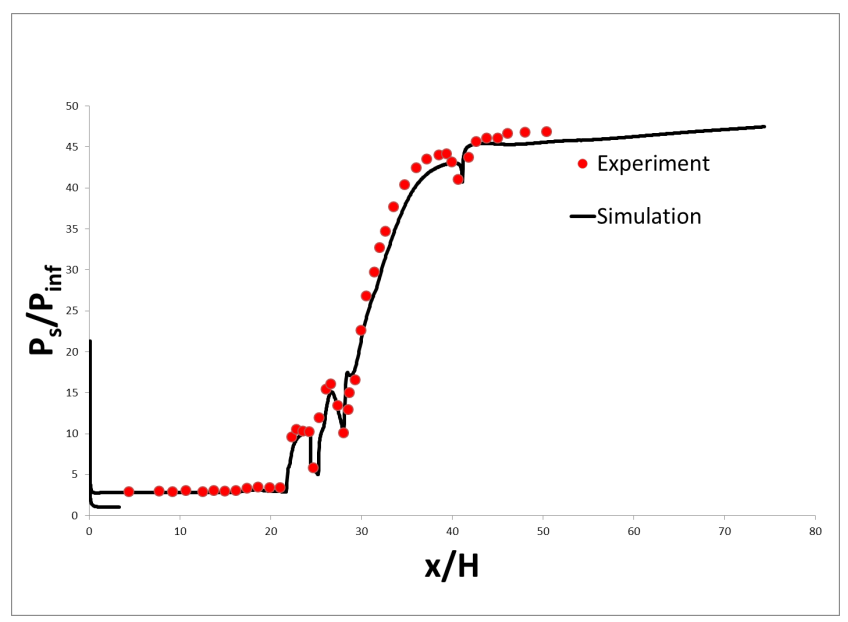

Figure 6: Supersonic Inlet/Isolator Mid-Plane, Body Side Static Pressure Comparison

and cowl induced shocks are clearly observed. The low Mach region observed near the leading edge of the cowl is due to the interaction of the shock waves from the side walls of the domain. The cowl shock impinges on the body-side boundary layer, inducing a low momentum region. This shock undergoes numerous reflections in the isolator region, terminating approximately near the middle of the isolator section. Corner vortices are observed near the cowl/side-wall intersections. The body-side flow separation is highlighted using streamlines. Downstream of the isolator, the diffuser induces large scale flow separation. Figure 6 is a plot of the mid-plane, body-side static pressure comparison between experimental data and current simulation results.

Accurately capturing the complicated shock structures and flow separation required a high resolution mesh, which is expensive to solve both in computation time and system resources. The simulation shown required 2500 fine grid iterations to obtain convergence, solved on 16 Titan nodes at a rate of 3.0 seconds per iteration. Each Titan node contains an AMD Opteron CPU and a single NVIDIA Tesla K20 accelerator. Without the use of the NVIDIA accelerator the solution time grew to 7.7 seconds per iteration. Thus, GPU acceleration with OpenACC directives has more than halved both the time to solution and the resource usage, with the Titan usage for this single computation decreased by over 53

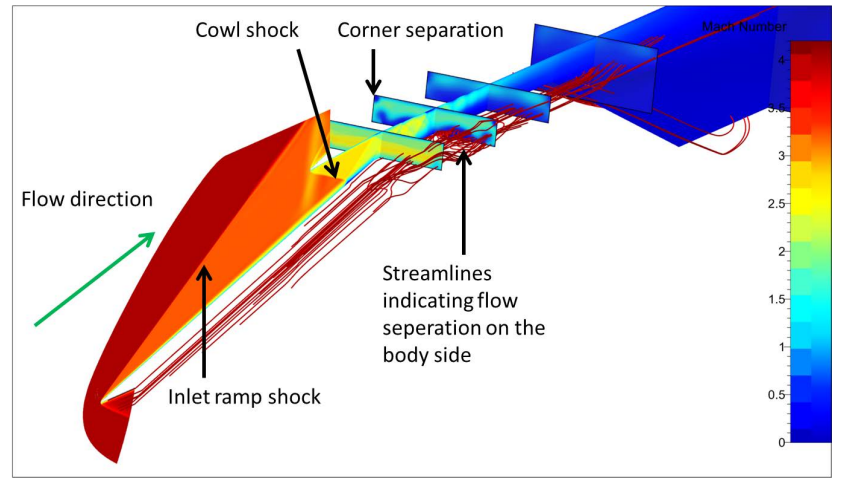

Figure 7: Supersonic Inlet/Isolator Mach Contour

node-hours. In practice, a product design cycle may require hundreds or even thousands of CFD simulations, and the computation time and system resource savings from GPU acceleration are magnified. It is worth re-iterating that the GPU acceleration did not impact the final solution or convergence behavior, with the accelerated and non-accelerated computations yielding identical results.

\section{CONCLUSIONS AND FUTURE WORK}

The successful adaptation of the NUMECA FINE/Turbo CFD solver for use in a heterogeneous CPU/GPU environment has been presented. The nature of industrial software development necessitates a quick, incremental development process, user-friendliness, and portability. An incremental acceleration through instrumentation of high value, end of call tree routines with OpenACC directives has been shown to be a feasible approach that meets these goals. The importance of nested data regions for improved data locality and an automatic acceleration management class for simplified performance tuning has also been shown. With these innovations, made possible due to the flexibility of OpenACC directives, an application speedup of over $2 \mathrm{X}$ has been obtained.

The AccManager auto tuning class has been shown to be useful as currently implemented, but additional development efforts may yield significant performance improvements. Future work will deeply analyze the runtime behavior of this class leading to improvements in the handling of multiple MPI processes. Also, the current work has focused on system configurations with a single accelerator. With minor modifications the AccManager should be able to auto-tune configurations with multiple accelerators per node.

\section{ACKNOWLEDGMENTS}

This research used resources of the Oak Ridge Leadership Computing Facility at the Oak Ridge National Laboratory, which is supported by the Office of Science of the U.S. Department of Energy under Contract No. DEAC0500OR22725. 


\section{REFERENCES}

[1] Emami, S., Trexler, C., Auslender, A., And WEIDNER., J. Experimental investigation of inlet-combustor isolators for a dual-mode scramjet at a mach number of 4. NASA Technical Paper 3502.

[2] Grosvenor, A., Rixon, G., Sailer, L., Matheson, M., Gutzwiller, D., Demeuleneare, A., Gontier, M., AND STrazisar, A. High resolution rans nonlinear harmonic study of stage 67 tip injection physics. Journal of Turbomachinery 137, 5 (MAY 2015).

[3] Grosvenor, A., Zheltovodov, A., Mathethon, M., SAiler, L., Krzysztopik, M., And Gutzwiller, D. Numerical analysis of three complex three-dimensional shock wave / turbulent boundary layer interaction flows. In EUCASS Book Series on Advances in Aerospace Sciences, Progress in Flight Physics, P. Reijasse, D. Knight, M. Ivanov, and I. Lipatov, Eds. EDP Press, 2013.

[4] Gutzwiller, D., And Gontier, M. Adaptation of a multi-block structured solver for effective use in a massively parallelized environment, May 2012. Presentation at the 24th International Conference on Parallel Computational Fluid Dynamics.

[5] Jameson, A., Schmidt, W., And Turkel, E. Numerical solutions of the euler equations by finite volume methods using runge-kutta time-stepping schemes. In AIAA Fluid and Plasma Dymanics
Conference Proceedings (1981). AIAA-81-1259.

[6] LARKIN, J. S4167: Introduction to accelerated computing using directives, March 2015. Presentation at the NVIDIA GPU Technology Conference.

[7] Lin, J., And Matsuoka, S. S4595: Openacc vs openmp4. the strong, the weak, and the missing to develop performance portable applications on gpu and the xeon phi, March 2014. Presentation at the NVIDIA GPU Technology Conference.

[8] NUMECA International. Numeca cpu-booster: Unique convergence acceleration technique. www.numeca.com/ en/ products/ finetmturbo/ cpuboostertm. Accessed: 2015-07-24.

[9] OpenaCC Standard Committee. The openacc application programming interface, version 2.0. Standard document, OpenACC-standard.org, June 2013.

[10] OpenMP4 Standard Committee. Openmp application program interface, version 4.0. Standard document, OpenMP.org, July 2013.

[11] Thebault, L., Petit, E., Tchiboukdjian, M., Dinh, Q., AND JalBy., W. Divide and conquer parallelization of finite element method assembly. In Parallel Computing: Accelerating Computational Science and Engineering, M.Bader, A.Bode, H.-J. Bungartz, M. Gerndt, G. Joubert, and F. Peters, Eds. IOS Press, 2014. 